\title{
Características maternas y resultados perinatales en mujeres peruanas infectadas con COVID-19:Un estudio observacional y transversal
}

\section{Maternal characteristic and perinatal outcomes among peruvian women infected with COVID-19. An observational and cross-sectional study}

Jorge A. Huatuco-Hernández ${ }^{1, \mathrm{a}, \mathrm{f}}$, Fredy J. Paredes-Villanueva ${ }^{1, \mathrm{a}, \mathrm{d}}$, Mick I. Quispe-Cuestas ${ }^{1, \mathrm{a}, \mathrm{f}}$, Germán A. Fiestas-Pflücker ${ }^{1, a, f}$, Maritza Nuñez-Rodas ${ }^{1, a, f}$, Xanadú Salazar-Cuba, ${ }^{1, c, h}$, Betty M. Zevallos-Vargas ${ }^{1, b, g}$, Amalia G. Vega-Fernández ${ }^{1, b, d}$, Robert P. Angeles-Reyes ${ }^{1, c, g}$, Julio Hilario-Vargas ${ }^{2, e}$

https://doi.org/10.35434/rcmhnaaa.2021.143.1276

\section{RESUMEN}

Objetive: Determinar las características maternas de mujeres infectadas con COVID-19 y de sus neonatos nacidos en el Hospital de atención referencia Covid-19 (HRDT) entre abril y setiembre del 2020. Material y Métodos: Estudio observacional y transversal realizado en el HRDT que incluyó 703 pacientes obstétricas con COVID-19. Resultados: La edad promedio fue de 27 años. El $35.7 \%$ fueron nulíparas y el 95\% fueron asintomáticas para COVID-19. Tuvieron COVID-19 leve, moderado y severo el $3 \%, 1 \%$ y $1 \%$; respectivamente. Los síntomas más frecuentes fueron tos $(84,85 \%)$ y anosmia $(39,39 \%)$. Los signos más comunes fueron Taquipnea $(60.61 \%)$ y dificultad respiratoria (51,52\%). La gasometría, dímero-D y ferritina sérica estuvieron alterados en el $71 \%, 54 \%$ y $30 \%$; respectivamente. Las complicaciones médicas más frecuentes fueron: sepsis $(2,28 \%)$, neumonía $(2,13 \%)$, insuficiencia respiratoria $(2,13 \%)$ y shock séptico $(1,14 \%)$. Las complicaciones obstétricas más frecuentes fueron Rotura prematura de membranas $(6,69 \%)$, preeclampsia severa $(5,41 \%)$, síndrome abortivo (3.27\%) y trabajo de parto pretérmino $(2,56 \%)$. El $73,9 \%$ de partos fue por Cesárea. El $0,99 \%$ de pacientes requirió unidad de cuidados intensivos y la letalidad materna fue del $0,2 \%$. El $85,7 \%$ de neonatos fueron a término y el $97,2 \%$ tuvo APGAR a los 5 minutos $\geq 7$. Hubo 4 muertes neonatales $(0,64 \%)$ y 16 óbitos $(2,58 \%)$. Hubo 8 RT-PCR positivas en neonatos $(1,3 \%)$. Conclusión: La mayoría de pacientes fueron nulíparas, menores de 35 años y asintomáticas para COVID-19. La mayoría de neonatos nacieron por cesárea y evolucionaron favorablemente. Hubo dos muertes maternas, 16 óbitos fetales y 4 muertes neonatales.

Palabras Clave: COVID-19, gestación, neonato (Fuente: DeCS-BIREME).

\section{ABSTRACT}

Objetive: To determine the maternal characteristics of women infected with COVID-19 and their neonates
FILIACIÓN

1. Hospital Regional Docente de Trujillo (HRDT). Trujillo, Perú.

2. Universidad Nacional de Trujillo. Trujillo, Perú.

a. Médico especialista en ginecología y obstetricia.

b. Médico subespecialista en neonatología

c. Médico residente en ginecología y obstetricia

d. Doctor en medicina.

e. Doctor en ciencias biomédicas

f. Maestro en medicina.

g. Maestro en gestión de servicios de salud

h. Bachiller en medicina.

ORCID

1. Jorge A. Huatuco-Hernández / 0000-0003-2986-6708

Fredy J. Paredes-Villanueva / 0000-0000-27638-0180

Mick I. Quispe-Cuestas /0000-0002-3518-2946
GermánA. Fiestas-Pflücker /0000-0003-1391-9596

Maritza Nuñez-Rodas / $\underline{0000-0001-6976-6766}$

CORRESPONDENCIA

Autor corresponsal: Jorge A. Huatuco Hernández. Av. Juan J. Ganoza 465. Dpto. 502, Urbanizaciónón California, Distrito de Víctor Larco, Trujillo-Perú. Código postal 13009.

EMAIL

jorgehuatuco1@gmail.com

CONFLICTOS DE INTERÉS

Los autores niegan conflictos de interés.

FINANCIAMIENTO

Autofinanciamiento.

CONTRIBUCIÓN DE LOS AUTORES

Jorge A. Huatuco-Hernández, Fredy Jesús Paredes-Villanueva, Mick Isaías Quispe-Cuestas, Germán Adolfo FiestasPflücker, Maritza Núñez-Rodas, Xanadú Salazar -Cuba, Betty M. Zevallos-Vargas, Amalia G. Vega Fernández, Robert P. aprobación de la versión final.

Además Jorge A. Huatuco- Hernández, Fredy Jesís Paredes-Vilnán Adolfo Fiestas-Pflücker, Maritza Núñez-Rodas, Xanadú Salazar -Cures-Villanueva, Mick Isaías Quispe-Cuestas, Germán Adolfo maternos y realizaron el análisis de datos maternos y Betty $M$. Zevallos-Vargas y Amalia $G$. Vega Fernández desarrollaron la base de datos neonatales y realizaron el análisis de datos del neonato. Julio Hilario-Vargas realizó la revisión final del artículo finalmente enviado.

\section{REVISIÓN DE PARES}

Recibido: 03/05/2021

Aceptado: 20/09/2021

COMO CITAR

Huatuco-Hernández, J., Paredes-Villanueva, F., Quispe-Cuestas, M., Fiestas-Pflücker, G., NuñezRodas, M., Salazar-Cuba, X., Zevallos-Vargas, B., Vega-Fernández, A., Angeles-Reyes, R., \& HilarioVargas, J. Caracteristicas maternas y resultados perinatales en mujeres peruanas infectadas con COVID-19: Un estudio observacional y transversal. Revista Del Cuerpo Médico Hospital Nacional A I m a n zor A g u in a g a A s e $\mathrm{j}$ jo, 2021,14 (3), 344 - 351 . https://doi.org/10.35434/rcmhnaaa.2021.143.1276

\section{(i)}

Esta obra está bajo una Licencia Creative Commons Atribución 4.0 Internacional.

Versión Impresa: ISSN: 2225-5109

Versión Electrónica: ISSN: 2227-4731

Cross Ref. DOI: $10.35434 /$ rcmhnaaa

OJS: https://cmhnaaa.org.pe/ojs 
born in the COVID-19 referral hospital (HRDT) between April and September 2020. Material and Methods: Observational and cross-sectional study carried out in the HRDT that included 703 obstetric patients with COVID-19. Results: The average age was 27 years. $35.7 \%$ were nulliparous and $95 \%$ were asymptomatic for COVID-19. 3\%, $1 \%$ and $1 \%$ had mild, moderate and severe COVID-19; respectively. The most frequent symptoms were cough $(84.85 \%)$ and anosmia $(39.39 \%)$. The most common signs were tachypnea $(60.61 \%)$ and respiratory distress (51.52\%). Blood gas, D-dimer, and ferritin were altered in $71 \%$, 54\%, and 30\%; respectively. The most frequent medical complications were: sepsis $(2.28 \%)$, pneumonia $(2.13 \%)$, respiratory failure $(2.13 \%)$ and septic shock $(1.14 \%)$. The most frequent obstetric complications were premature rupture of membranes $(6.69 \%)$, severe preeclampsia $(5.41 \%)$, abortive syndrome $(3.27 \%)$ and preterm labor $(2.56 \%)$. $73.9 \%$ of deliveries were by Caesarean section. $0.99 \%$ of patients required intensive care unit and maternal mortality was $0.2 \% .85 .7 \%$ of neonates were at term and $97.2 \%$ had APGAR $\geq 7$ at 5 minutes. There were 4 neonatal deaths $(0.64 \%)$ and 16 deaths $(2,58 \%)$. There were 8 positive RTPCR in neonates (1.3\%). Conclusion: The majority of patients were nulliparous, under 35 years of age and asymptomatic for COVID-19. Most of the newborns were born by cesarean section and evolved favorably. There were two maternal deaths, 16 stillbirths, and 4 neonatal deaths

Keywords: COVID-19, pregnancy, newborn (Source: DeCS-BIREME).

\section{INTRODUCCIÓN}

El COVID-19 es una patología de gran importancia epidemiológica en el contexto actual debido a la rápida propagación del virus y al hecho que los brotes pueden crecer a un ritmo exponencial ${ }^{(1)}$; sumado a la presencia de complicaciones graves que aumentan la morbimortalidad tales como neumonía, SIRS, sepsis, RITAC y falla multiorgánica, seguidas de muerte ${ }^{(2)}$.

La propagación mundial del virus ha desbordado los sistemas sanitarios y ha provocado una amplia perturbación social y económica. La pandemia exige un alto precio a personas, familias, comunidades y sociedades de todo el mundo ${ }^{(1)}$.

La trayectoria de este brote es imposible de predecir hoy en día, pero parece evidente que para alcanzar una respuesta efectiva se requiere una acción rápida desde el punto de vista de las estrategias clásicas de salud pública ${ }^{(3)}$. Dentro de estas estrategias se ha propuesto el distanciamiento social, el uso de mascarillas, el lavado de manos y actualmente nos encontramos en la primera fase de aplicación de la vacuna, la cual comenzó a aplicarse en nuestro país en febrero del 2021, priorizando al personal de salud ${ }^{(4)}$.

Al día 14 de setiembre 2020, fecha de actualización usada, se registró la siguiente estadística: a nivel mundial la incidencia de COVID-19 fue de 28,871,176 infectados ${ }^{(5)}$. El Ministerio de Salud de Perú reportó para esa fecha un total de 733,860 ciudadanos infectados y 30,812 fallecidos, con una tasa de letalidad de 4,19\% ${ }^{(6)}$. En la región La Libertad desde el inicio de la pandemia se han detectado 48,088 casos con 3420 fallecidos $^{(7)}$.

En la actualidad, en relación con el efecto de esta pandemia en el embarazo se tienen más preguntas que certezas acerca del real impacto del COVID-19 en gestantes. Los estudios se actualizan y muchas veces se contradicen.

Al momento de finalizar el presente estudio, no existe evidencia que sugiera que las gestantes con COVID-19 presenten mayor morbilidad que las mujeres no embarazadas afectadas $^{(8)}$.
Sin embargo, aún no se han estudiado un número suficiente de gestantes que permita realizar inferencias apropiadas. Se conoce que la infección por SARS O MERS aumentan significativamente el número de muertes maternas, pero esto no parece ser cierto para el caso de COVID-19, pero si al parecer incrementa la tasa de parto prematuro, preeclampsia, cesárea y muerte perinatal. Sin embargo, aún no se tiene información suficiente para afirmar que no existe infección vertical en estas gestantes ${ }^{(9)}$.

El Hospital Regional Docente de Trujillo (HRDT) fue designado hospital COVID exclusivo desde el inicio de la emergencia sanitaria declarada en nuestro país, a partir del 14 de marzo del 2020 y se tuvo que acondicionar y adaptar ambientes, recursos humanos, recursos logísticos y procesos para enfrentar esta amenazante y letal enfermedad.

En el presente reporte se presentan las características de gestantes, parturientas y puérperas infectadas con COVID-19 y de sus neonatos nacidos en el Hospital Regional Docente de Trujillo; Perú, en el periodo de abril a setiembre del 2020; a fin de contribuir a conocer mejor el comportamiento del COVID-19 durante la primera ola.

\section{MATERIALY MÉTODOS}

Se llevó a cabo un estudio observacional y transversal que incluyó 703 gestantes y puérperas internadas en el Hospital Regional Docente de Trujillo-Perú, hospital referencial COVID-19, de la Región La Libertad, en el periodo de estudio (del 18/04 al 14/09 del año 2020) y que tuvieron prueba serológica o molecular positiva para COVID-19. Se utilizó como primer criterio de diagnóstico de COVID-19 la serología positiva, debido a que, en el periodo de estudio, las pruebas serológicas fueron las más accesibles en los establecimientos de atención del Ministerio de Salud del Perú. La prueba molecular positiva, debido a su costo, se utilizó para el diagnóstico de COVID-19 en pacientes con sintomatología compatible, pero con serología negativa.

De nuestra base de datos inicial que incluía a 717 pacientes, fueron excluidas 14 pacientes, que no tenían una condición obstétrica o eran casos ginecológicos. Entre ellas, una se 
excluyó por no llegarse a confirmar el diagnóstico COVID-19 (prueba serológica y PCR Negativo) y otra paciente que fue ingresada como aborto incompleto después se confirmó que era un cáncer de endometrio.

De las 703 pacientes obstétricas, $619(88,1 \%)$ tuvieron parto en el HRDT y $84(11,9 \%)$ llegaron como puérperas.

Los datos para la elaboración del presente estudio fueron obtenidos a partir de dos bases de datos diseñadas previamente: base de datos de gestantes y puérperas con COVID-19 (desarrollado por el Departamento de Ginecología y Obstetricia del HRDT) y base de datos de neonatos de madres con COVID-19 (desarrollado por el Departamento de Pediatría del HRDT).

Para la elaboración del presente estudio se contó con el permiso correspondiente de la Dirección del HRDT y durante su desarrollo se cumplió con una sólida base ética fundamentada en los principios establecidos en la Declaración de Helsinki, relacionándose directamente con los principios 9 y 24, los cuales puntualizan la confidencialidad, responsabilidad y el resguardo de la intimidad del paciente en la ejecución del estudio ${ }^{(10)}$.

\section{Criterios de inclusión:}

- Gestantes o puérperas con diagnóstico de COVID-19 evidenciado mediante cualquiera de los siguientes resultados: Serología positiva para COVID-19. Se consideró cualquiera de los siguientes resultados: IgM, IgG o lgM/lgG positivos; o Prueba molecular TC-PCR positiva.

\section{Criterios de exclusión:}

- Aquellas que no tuvieron registro de datos clínicos o de laboratorio pertinentes para ser incorporadas en el estudio.

- Pacientes que presentaron diagnostico presuntivo de COVID-19 y no confirmado posteriormente como COVID19 por PCR-RT o serología.

Los datos de las historias clínicas de las pacientes fueron transcritos en una base de datos (hoja de cálculo Excel), creada para tal fin y analizados descriptiva y gráficamente, utilizando metodológicamente la estadística descriptiva para variables de posición y de variación.

\section{RESULTADOS}

En el periodo comprendido entre el 18 de abril al 14 de septiembre ingresaron a nuestro hospital 717 pacientes al Departamento de ginecoobstetricia COVID del HRDT. De estas fueron eliminadas del presente estudio 14 pacientes, una por descartarse el COVID-19 luego de PCR-RT y prueba serológica, otra por tener cáncer de endometrio y las restantes por presentar diagnósticos ginecológicos.

En la tabla 1 se observa: El promedio de edad fue de 27 años. El $83,2 \%$ tuvieron menos de 35 años, el $35,7 \%$ fueron nulíparas. Respecto a la procedencia el $76,7 \%$ pertenecen a la provincia de Trujillo.
Tabla 1. Características epidemiológicas de pacientes obstétricas hospitalizadas por COVID-19 en el HRDT, Trujillo-Perú. Abril a Septiembre del 2020.

\begin{tabular}{lcc} 
Edad $(\mathrm{x}, \mathrm{s})$ & 27 & \pm 7.09 \\
Edad $\geq 35(\mathrm{n} / \mathrm{N}, \%)$ & $114 / 703$ & 0,162 \\
Paridad $(\mathrm{n} / \mathrm{N}, \%)$ & $\mathrm{n} / \mathrm{N}$ & $\%$ \\
Nulípara & $251 / 703$ & 35,7 \\
Primípara & $237 / 703$ & 33,7 \\
Multipara & $215 / 703$ & 30,5 \\
\hline
\end{tabular}

En la tabla 2 se consigna lo siguiente: El 95\% fueron asintomáticas para COVID-19. El 3,1 y $1 \%$ tuvieron COVID leve, moderado y severo; respectivamente. En las pacientes sintomáticas: tos, frecuencia respiratoria > de 30 , dificultad respiratoria, anosmia y fiebre, fueron los síntomas o signos más comunes, con $75,6 \%, 54 \%, 45,9 \%, 35,1 \%, 18,9 \%$; respectivamente.

Tabla 2. Características clínicas de pacientes obstétricas hospitalizadas por COVID-19 en el HRDT, Trujillo-Perú. Abril a Septiembre del 2020.

\begin{tabular}{lcc} 
Signos y síntomas & $\mathrm{n}$ & $\%$ \\
Tos & 28 & 84,85 \\
Frecuencia respiratoria $>30$ & 20 & 60,61 \\
Dificultad respiratoria & 17 & 51,52 \\
Presión sistólica $<100$ & 16 & 48,48 \\
Saturación de oxígeno $<90 \%$ & 14 & 42,42 \\
Anosmia & 13 & 39,39 \\
Fiebre & 7 & 21,21 \\
Escala de Glasgow $\leq$ de 13 & 6 & 18,18 \\
Náuseas y vómitos & 4 & 12,12 \\
Diarrea & 2 & 6,06 \\
\hline
\end{tabular}

En la tabla 3 se consigna que los exámenes de laboratorio que con más frecuencia estuvieron alterados fueron: gasometría, dímero-D, ferritina y hemoglobina con $71,4 \%, 54,8 \%, 30,4 \%$ y $25,8 \%$; respectivamente.

Tabla 3: Variables de laboratorio de pacientes obstétricas hospitalizadas por COVID-19 en el HRDT, Trujillo-Perú. Abril a Septiembre del 2020.

\begin{tabular}{lcc}
\hline Recuento leucocitario ( , s) & 10671,49 & 3947,64 \\
$<4,000$ /uL (n/N,\%) & $6 / 529$ & $1,13 \%$ \\
$>15,000 / \mathrm{LL}(\mathrm{n} / \mathrm{N}, \%)$ & $47 / 529$ & $8,88 \%$ \\
Proteína C reactiva mg/L( , s) & 22,61 & 36,92 \\
$>100(\mathrm{n} / \mathrm{N}, \%)$ & $4 / 135$ & $2,96 \%$ \\
Recuento de plaquetas ( , s) & 239797 & 74757 \\
$<150,000 / \mathrm{ml}(\mathrm{n} / \mathrm{N}, \%)$ & $55 / 527$ & $4,74 \%$ \\
Hemoglobina <10 al ingreso (n/N , \%) & $170 / 658$ & $25,84 \%$ \\
Perfíl hepático alterado(n/N,\%) & & \\
TGO & $20 / 509$ & $3,93 \%$ \\
TGP & $12 / 508$ & $2,36 \%$ \\
Creatinina sérica>1,2mg/dl(n/N,\%) & $4 / 500$ & $0,8 \%$ \\
Ferritina sérica ng/ml ( , s) & 128,29 & 180 \\
Ferritina sérica>100 (n/N , \%) & $7 / 23$ & $30,43 \%$ \\
Ferritina sérica>300 (n/N , \%) & $4 / 23$ & $4,3 \%$ \\
Dímero D ug/L ( , s) & 1392 & 1008 \\
Dímero D >1000 (n/N , \%) & $17 / 31$ & $54,83 \%$ \\
Dímero D >1700 (n/N , \%) & $10 / 31$ & $32,25 \%$ \\
Gasometría alterada(n/N,\%) & $10 / 14$ & $71,4 \%$ \\
PaO2<70 mmHg(n/N,\%) & $10 / 14$ & $71,4 \%$ \\
PaO2/FiO2<300(n/N,\%) & $10 / 14$ & $71,4 \%$ \\
Lactato 2 mmol/L & $4 / 14$ & $28,6 \%$ \\
\hline
\end{tabular}

Tabla 2. Características clínicas de pacientes obstétricas hospitalizadas por COVID-19 en el HRDT, Trujillo-Perú. Abril a Septiembre del 2020. 
En la tabla 4 se detalla que las complicaciones médicas más frecuentes fueron: sepsis, neumonía, insuficiencia respiratoria y shock séptico con $2,2 \%, 2,1 \%, 2,1 \%$ y $1,1 \%$; respectivamente.

Tabla 4. Complicaciones médicas en pacientes obstétricas hospitalizadas por COVID-19 en el HRDT, Trujillo-Perú. Abril a Septiembre del 2020 (703 pacientes).

\begin{tabular}{lcc} 
Complicaciones relacionadas al COVID-19 & $\mathrm{n}$ & $\%$ \\
Sepsis (Quick SOFA) & 16 & 2,28 \\
Neumonía & 15 & 2,13 \\
Insuficiencia respiratoria* & 15 & 2,13 \\
Shock séptico & 8 & 1,14 \\
Falla orgánica multiple & 6 & 0,85 \\
Sindrome RITAC & 6 & 0,14 \\
Injuria renal aguda & 5 & 0,71 \\
Otro & & \\
\hline${ }^{*}$ Incluyó insuficiencia respiratoria leve, moderada y severa &
\end{tabular}

En la tabla 5 se observa que los diagnósticos obstétricos más frecuentes fueron; Ruptura prematura de membranas, preeclampsia severa, síndrome abortivo, trabajo de parto pre término con $6,69 \%, 5,41 \%, 3,27 \%$ y $2,56 \%$; respectivamente.

Tabla 5. Diagnósticos obstétricos de pacientes hospitalizadas por COVID-19 en el HRDT, Trujillo-Perú. Abril a Septiembre del 2020 (703 pacientes).

\begin{tabular}{lcc} 
Diagnósticos obstétricos & $\mathrm{n}$ & $\%$ \\
Gestante a término & 393 & 55,9 \\
Labor de parto a término & 116 & 16,5 \\
Rotura prematura de membranas & 47 & 6,69 \\
Otro trastorno hipertensivo & 46 & 6,54 \\
Preeclampsia severa & 38 & 5,41 \\
Labor de parto a pretérmino & 18 & 2,56 \\
Sindrome abortivo & 27 & 3,84 \\
Embarazo ectópico & 7 & 0,99 \\
Sufrimiento fetal & 5 & 0,71 \\
Amenaza de parto prematuro & 5 & 0,71 \\
Otros & 1 & 0,15 \\
\hline
\end{tabular}

En la tabla 6 y 7 se observa que del total de puérperas que salieron de alta el $88 \%$ tuvieron parto por cesárea o parto vaginal (hubo pacientes que fueron referidas a nuestro hospital ya en estado de puérperas). Del total de partos atendidos en nuestra institución el 73,9\% fue por cesárea. El $0,99 \%$ de pacientes requirió internamiento en unidades de cuidados críticos ( $\mathrm{UCI}$ ) y la tasa de letalidad fue del $0,2 \%$.

Tabla 6. Condición obstétrica al alta de pacientes hospitalizadas por COVID-19 en el HRDT, Trujillo-Perú. Abril a Septiembre del 2020 (703 pacientes).

\begin{tabular}{lcc}
\hline Puérperas (n, \%) & 636 & $88 \%$ \\
Cesárea (n, \%) & 458 & $73,9 \%$ \\
Parto vaginal (n, \%) & 161 & $26.00 \%$ \\
Puérpera referida* & 17 & $2,4 \%$ \\
Pérdida gestacional en la primera mitad del embarazo (n,\%) & 34 & $4,8 \%$ \\
Gestantes (n, \%) & 33 & $4,69 \%$ \\
\hline
\end{tabular}

*Pacientes con parto en otra institución que fueron referidas al HRDT.

Tabla 7. Evolución y Desenlace de pacientes obstétricas hospitalizadas por COVID-19 en el HRDT, Trujillo-Perú. Abril a Septiembre del 2020 (703 pacientes).

\begin{tabular}{lcc}
\hline Ingreso a unidades de cuidados críticos (n, \%) & 7 & $0,99 \%$ \\
UCI & 4 & $0,56 \%$ \\
UCIM-Trauma shock & 3 & $0,43 \%$ \\
Alta hospitalaria $(\mathrm{n}, \%)$ & 701 & $99,7 \%$ \\
Muerte hospitalaria $(\mathrm{n}, \%)$ & 2 & $0,28 \%$ \\
Muerte domiciliaria $(\mathrm{n}, \%)$ & 0 & 0 \\
\hline
\end{tabular}

En la tabla 8 se detalla: De los 613 recién nacidos atendidos, los resultados perinatales más frecuentes fueron edad gestacional $\geq 37$ semanas, en un $14,3 \%$, con prematuros moderados en un $11,4 \%$ entre $\geq 34-36$ semanas. La patología más frecuente y causa de hospitalización fue la sepsis neonatal con $10,3 \%$. Se tuvo 3 muertes neonatales tempranas, todas ellas en prematuros entre 24 a 34 semanas de edad gestacional, las causas de muerte no fueron atribuidas a COVID-19. Y se presentó un caso de muerte neonatal tardía en un prematuro de 25 semanas de edad gestacional quien tuvo como causa básica de muerte enterocolitis necrotizante. A todos los neonatos se les realizó PCR-RT teniendo resultados positivos en 8 casos $(1,3 \%)$.

Tabla 8. Características perinatales del producto de pacientes obstétricas hospitalizadas por COVID-19 en el HRDT, Trujillo-Perú. Abril a Setiembre del 2020 (613 pacientes).

\begin{tabular}{|c|c|c|}
\hline \multirow[t]{2}{*}{ Edad gestacional por examen físico: $\left(\mathrm{x}^{-}, \mathrm{s}\right)$} & \multicolumn{2}{|l|}{$38,3 \pm 2,1$} \\
\hline & $\mathrm{n}$ & $\%$ \\
\hline$<28$ semanas & 2 & 0,3 \\
\hline $28-33$ semanas & 16 & 2,6 \\
\hline $34-36$ semanas & 70 & 11,4 \\
\hline$\geq 37$ semanas & 525 & 85,6 \\
\hline \multirow[t]{2}{*}{ Peso $\left(x^{-}, s\right)$} & \multicolumn{2}{|l|}{$3212 \pm 582,5$} \\
\hline & $\mathrm{n}$ & $\%$ \\
\hline$<1000$ gramos & 1 & 0,2 \\
\hline $1000-<1500$ gramos & 3 & 0,5 \\
\hline $1500-<2500$ gramos & 57 & 9,3 \\
\hline$\geq 2500$ gramos & 552 & 90 \\
\hline Apgar a 1 minuto & $\mathrm{n}$ & $\%$ \\
\hline$\leq 3$ & 5 & 0,8 \\
\hline 04-jun & 12 & 2 \\
\hline$\geq 7$ & 596 & 97,2 \\
\hline Apgar a 5 minuto & $\mathrm{n}$ & $\%$ \\
\hline$\leq 3$ & 1 & 0,2 \\
\hline 04-jun & 2 & 0,3 \\
\hline$\geq 7$ & 610 & 99,5 \\
\hline Complicaciones del neonato: hospitalizados ( $\mathrm{n}, \%$ ) & 125 & 20,4 \\
\hline Sepsis & 63 & 10,3 \\
\hline Ictericia & 13 & 2,1 \\
\hline Enfermedad de membrana hialina & 10 & 1,6 \\
\hline Taquipnea transitoria del RN & 6 & 1 \\
\hline PEG severo & $8 /$ & 1,3 \\
\hline Neumonía congénita & 4 & 0,7 \\
\hline SALAM & 4 & 0,7 \\
\hline Asfixia perinatal & 1 & 0,2 \\
\hline Otra morbilidad & 16 & 2,6 \\
\hline Condición de egreso & $\mathrm{n}$ & $\%$ \\
\hline Alta & 609 & 99,3 \\
\hline Fallecido & 4 & 0,7 \\
\hline Muerte perinatal * & $n$ & $\%$ \\
\hline Óbito fetal & $16 / 619$ & 2,58 \\
\hline Muerte neonatal precoz & $3 / 619$ & 0,48 \\
\hline Muerte neonatal tardía & $1 / 619$ & 0,16 \\
\hline PCR-RT positiva & $8 / 613$ & 1,3 \\
\hline
\end{tabular}


Revista del Cuerpo Médico del HNAAA, Vol 14 (3) - 2021

Sobre las características de los neonatos con PCR-RT positiva para SARS-CoV-2, en el HRDT, Trujillo-Perú, se encontró 2 neonatos por vía vaginal y 6 vía por cesarea, respecto a la severidad, 6 fueron asintomáticos y solo dos presentaron grado leve, y todos los neonatos fueron dados de alta vivos. Hubo diversidad de tiempo para la toma de prueba post natal, variando de menos de 10 minutos (4 neonatos), de 50 minutos (1 neonato), de 85 minutos (2 neonatos) y 120 minutos (1 neonato).

Tabla 9. Óbitos fetal en pacientes Hospitalizadas por COVID-19 en el HRDT, Trujillo-Perú. Abril a Septiembre del 2020 (16 pacientes).

\begin{tabular}{lcc} 
Características de las madres y óbitos & $\mathrm{n}$ & $\%$ \\
Momento de la muerte & & \\
Extrahospitalaria & 10 & $62,5 \%$ \\
$\quad$ Intrahospitalaria & 6 & $37,5 \%$ \\
Severidad Covid & & \\
Asintomática & 14 & $87,5 \%$ \\
Leve & 1 & $6,25 \%$ \\
Moderada & 0 & $0 \%$ \\
Severa & 1 & $6,25 \%$ \\
Patología materna /fetal & & \\
Sufrimiento Fetal Agudo & 3 & $18,75 \%$ \\
Malformación fetal & 2 & $12,5 \%$ \\
Corioamnionitis & 1 & $6,25 \%$ \\
Placenta previa & 1 & $6,25 \%$ \\
Rotura Uterina & 1 & $6,25 \%$ \\
RPM & 1 & $6,25 \%$ \\
Ninguna & 7 & $43,75 \%$ \\
Edad gestacional & & \\
21- 28 semanas & 4 & $25 \%$ \\
29-36 semanas & 5 & $31,25 \%$ \\
$>37$ semanas & 7 & $43,75 \%$ \\
\hline
\end{tabular}

\section{DISCUSIÓN}

\section{Resultados maternos:}

El presente estudio se ejecutó en el HRDT, teniendo como base una población de 703 pacientes obstétricas ingresadas con diagnóstico confirmado de COVID-19 por serología.

La mayor parte de pacientes estuvieron entre los 20 y 35 años y principalmente nulíparas. Estos datos son coincidentes con otros reportes como el de Huerta et $\mathrm{al}^{(11)}$. La mayor frecuencia de casos se presentó en el Trujillo urbano seguido de los distritos vecinos. Y a nivel provincial destaca la provincia de Chepén. La frecuencia de casos descrita se correlaciona estrechamente con la frecuencia de casos de COVID-19 en la población general ${ }^{(12)}$.

La frecuencia de síntomas en las gestantes en este reporte estuvo en proporción inversa a la frecuencia en la población en general. El 95\% (670/703) eran asintomáticas en comparación con el $29 \%$ reportado a nivel nacional ${ }^{(13)}$, pero mayor que un estudio previo en 41 pacientes obstétricas que reporta $68,2 \%{ }^{(11)}$. No obstante, la frecuencia de síntomas es ligeramente diferente con los primeros reportes de China, donde en sólo 9 gestantes el 77,7\% presentaron fiebre, seguido del $55,5 \%$ con tos ${ }^{(14)}$. Nosotros encontramos que los síntomas más frecuentes fueron tos $(84,85 \%)$ y anosmia $(39,39 \%)$ y los signos más comunes fueron Taquipnea $(60,61 \%)$ y dificultad respiratoria $(51,52 \%)$.
Esto ocurrió probablemente por el momento de la enfermedad en que se encontraban nuestras pacientes, a juzgar por los valores positivos tanto de lg M como de lg G hallados simultáneamente, lo cual indicaría que las pacientes estaban en la segunda semana de la enfermedad cuando el virus había invadido ya el tracto respiratorio bronquial.

Si bien, teóricamente el embarazo por razones inmunológicas y fisiológicas predispone a mayor probabilidad de infección respiratoria severa, en nuestra serie no se encontró una diferencia cuantitativa con la población no gestante. Nuestro grupo encontró que el $0,99 \%$ requirió ingreso a $\mathrm{UCl}$, muy similar al 4,8\% reportado por Huerta et $\mathrm{al}^{(11)}$. Sin embargo, nuestro hallazgo fue inferior al reportado por Lokken EM et

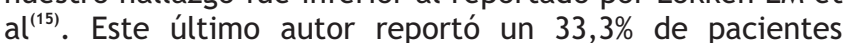
internados en $\mathrm{UCl}$. Las diferencias tan notables con este último estudio se deben al tipo de población incluida: en el estudio de Lokken todas las pacientes que ingresaron al estudio eran sintomáticas, a diferencia de nuestro estudio que incluyó en su gran mayoría pacientes asintomáticas que se internaron para terminar el embarazo.

Sin embargo, tuvimos dos casos con desenlace fatal. Este hallazgo podría indicar que aunque es igual la frecuencia de casos severos, el pronóstico suele ser peor, sobre todo en presencia de alguna comorbilidad, El primer caso de muerte materna tuvo preeclampsia severa y síndrome de HELLP y el segundo caso fue una paciente sin comorbilidad. Esto contrasta con el reporte de Liu et $\mathrm{al}^{\left({ }^{(16)}\right.}$ que en sólo 15 gestantes con neumonía por COVID-19 ninguna hizo un caso severo.

En los exámenes de laboratorio realizados se encontró un valor de leucocitos de $10671 / \mathrm{uL} \pm 3947 / \mathrm{uL}$, lo cual difiere del estudio de Jie Yan, et al ${ }^{(17)}$. realizado de manera retrospectiva en 25 hospitales de china en 116 pacientes obstétricas, donde obtuvieron una media de valor de leucocitos de 7900/UL; probablemente es debido a que en nuestro estudio presentamos 116 pacientes en labor de parto lo que implica un aumento de leucocitos fisiológico, a diferencia de dicho estudio donde describen labor de parto en menor cantidad (14 pacientes) ${ }^{(17)}$. A su vez en el estudio retrospectivo de $\mathrm{Xu}$ Qiancheng, et al., evaluó 28 gestantes y 54 mujeres en edad reproductiva no gestante, en el periodo de 15 de enero a 15 de marzo del presente año en el hospital central de Wuhan, donde se detectó mayor leucocitosis en pacientes gestantes $(10,35,7 \%)$ que en no gestantes $(2,3.7 \%)(p<0.001)$, lo cual es similar a nuestro estudio ${ }^{(18)}$.

El siguiente parámetro estudiado fue proteína C Reactiva, se encontró elevación de este en solo un 2,96\% de pacientes, un resultado diferente al estudio de Jie Yan, et al. En el que encontraron elevación de este parámetro en un $44 \%$ de las pacientes, debido probablemente a que la gran parte de nuestras pacientes fueron asintomáticas por lo que no tenían un cuadro inflamatorio sistémico que se traduzca en la elevación de este parámetro ${ }^{(17)}$.

El dímero D se encontró elevado en el 54,83\%. Dato similar al reportado por Xu Qiancheng, et al. ${ }^{(18)}$. Este autor describe que el 92,3\% de pacientes gestantes tuvieron elevación de Dímero D. Este evento se puede explicar porque el COVID-19 cursa con eventos de micro trombosis. El valor del dímero $D$ es 
relevante y es utilizado como criterios de severidad, pues evidenciamos en nuestro estudio la asociación entre Dímero D por encima de $1700 \mathrm{ug} / \mathrm{L}$ y síndrome de respuesta inmunológica y trombótica asociada al COVID-19 (RITAC).

Chen et $\mathrm{al}^{(19)}$ analizaron las características clínicas de 99 pacientes no gestantes, en los cuales 63 (63\%) presentaron ferritina sérica muy por encima de lo normal, algo que también encontramos en nuestras pacientes, 30,4\% de ellas presentaron una elevación de ferritina, que como se sabe mide el proceso inflamatorio y su severidad ${ }^{(19)}$.

Las lecciones aprendidas en relación con el H1N1 y SARS-CoV1 sugieren que las mujeres embarazadas son particularmente susceptibles a los resultados adversos, incluida la necesidad de intubación endotraqueal ${ }^{(20)}$, debido a razones inmunológicas y fisiológicas, no obstante, en nuestra serie no se encontró una diferencia cuantitativa con la población no gestante. Nuestro grupo encontró que el 0,99\% requirió ingreso a UCI. Cifra inferior a la reportada por Lokken, et $\mathrm{al}^{(15)}$ que estudio 240 casos confirmados de infecciones por SARSCoV-2 durante el embarazo de las cuales 3,3\% requirió ingreso a $\mathrm{UCl}$ y al reportado por Huerta et $\mathrm{al}^{(11)}$ en Lima-Perú en una población obstétrica con $4,8 \%$.

En nuestro estudio el 5,1\% de pacientes tuvieron COVID-19 Severo, cantidad menor a la encontrada por Lokken, et $\mathrm{al}^{\left({ }^{(15)} \text {. }\right.}$ Este autor encontró que 9,2\% de sus pacientes presentaron infección severa; sin embargo, al igual que ellos, nuestras pacientes también presentaron complicaciones mayores como falla multiorgánica, shock séptico, insuficiencia respiratoria grave con necesidad de ventilación invasiva, síndrome RITAC e insuficiencia renal.

En nuestro estudio tuvimos dos casos fatales, un $0.28 \%$, valor menor al encontrado por Lokken, et al ${ }^{(15)}$ que tuvieron $1,3 \%$; probablemente porque nuestra cantidad de paciente fue mayor y nuestro tiempo de estudio también lo fue. Sin embargo, concordamos en la fisiopatología de la causa de la muerte pues en la primera paciente con muerte materna fue derivada en estado de puerperio de otra institución en la que había sido operada por preeclampsia severa y se cree que en la causa de muerte existieron efectos aditivos como la disfunción endotelial asociada al trastorno hipertensivo del embarazo y la generada por el COVID. Tal como Mendoza et postula, indicando que el COVID severo puede simular procesos parecidos al síndrome de preeclampsia severa y síndrome de HELLP ${ }^{(14)}$.

La segunda muerte materna tenía una gestación no evolutiva de 21 semanas. No tenía co-morbilidades identificadas. Ingresó con insuficiencia respiratoria severa y falla renal llegándose a realizar hemodiálisis, permaneciendo varios días en $\mathrm{UCl}$, proceso similar a los eventos de muerte materna reportados por a Lokken, et $\mathrm{al}^{\left({ }^{(1)}\right)}$ : Su tercera paciente murió de insuficiencia respiratoria después de una estadía prolongada en la $\mathrm{UCl}$ a pesar de múltiples terapias contra el COVID-19.

No se ha evidenciado que la infección por SARS-CoV-2 durante el embarazo se asocie con un mayor riesgo de aborto espontaneo y parto prematuro espontaneo, sin embargo, algunas series publicadas describen una mayor incidencia de complicaciones durante el embarazo o el parto en mujeres afectadas por COVID. Schwartz et $\mathrm{al}^{(21)}$ en una serie de 38 embarazos, describen diabetes gestacional, pre eclampsia, rotura uterina, hipertensión gestacional e hipotiroidismo y en relación al parto, el feto o el neonato: nacimientos pre término, distrés fetal, rotura prematura de membranas, alteraciones del cordón umbilical, placenta previa, corioamnionitis, oligo y polihidramnios y líquido amniótico meconial.

Aunque la vía de parto se decide por indicaciones obstétricas usuales y la condición general de la paciente, es necesario comentar que tal decisión no debe ser estrictamente influenciada la presencia de la enfermedad ${ }^{(20,22,23)}$, en el HRDT se ha protocolizado la terminación de las gestaciones por la vía más rapida, incluída la cesárea, pero basada en indicaciones obstétricas principalmente, con la finalidad de disminuir el tiempo de exposición del personal de salud y a los aerosoles que se producen en el segundo periodo del parto. De otra parte, muchas pacientes fueron referidas a nuestro hospital, COVID específico, para culminación del embarazo, por antecedente de cesáreas previas lo que contribuyó a que los porcentajes de cesárea sean altos $(73,1 \%)$. Similar resultado fue obtenido por Huerta et $\mathrm{al}^{(11)}$ en Lima, Perú; en cuya estadísitica se reporta un 78,3\% de cesáreas.

\section{Resultados perinatales del neonato:}

En la Tabla 8 se muestran los resultados perinatales, de los 613 casos. El 85,6\% (525/613) fueron recién nacidos de término (RNT) y el $14,3 \%(88 / 613)$ fueron recién nacidos pretérminos (RNpT), el peso promedio al nacer fue de $3212 \mathrm{~g}$, $\pm 582,5$ g y el $10 \%(61 / 613)$ tuvieron bajo peso al nacer.

Estos datos que concuerdan con lo reportado Yang et $\mathrm{al}^{(23)}$ quienes reportan que la edad gestacional promedio fue de $38,2 \pm 2,3$ y el peso promedio fue $3063,2 \pm 536,4$, datos muy similares a los encontrados por Chen et al. ${ }^{(24)}$. Con estos resultados podemos evidenciar que la mayor parte de gestantes con COVID-19 puede llegar a una gestación a término y con peso al nacer dentro de valores de normalidad.

Sin embargo, Zhu et $\mathrm{al}^{(25)}$ y Di Mascio et $\mathrm{al}^{\left({ }^{(9)}\right.}$ encontraron una alta tasa de prematuros $60 \%$ y $41.1 \%$ respectivamente, y esto debido probablemente a que en los inicios de pandemia, al diagnosticarse COVID-19, se decida por término de gestación rápidamente.

Referente a la valoración de Apgar el 0,2\% (1/613) tuvieron $\leq$ 3 a los 5 minutos, con diagnóstico de asfixia perinatal. Chen et al. ${ }^{(24)}$ y $\mathrm{Yu}$ et $\mathrm{al}^{(26)}$ reportan que el total de los neonatos estudiados tuvieron Apgar al minuto y 5 minutos $\geq 7$ en ambos estudios. Por otro lado, Di Mascio et al..$^{(9)}$ encontraron que la tasa de puntuación de Apgar < 7 a los 5 minutos fue del 4,5\% (1 de 41 , IC del 95\%, 0,4-12,6), y no se notificó ningún caso de asfixia neonatal.

Se encontró que el 20,4\% (125/613) de los neonatos fueron ingresados al servicio de neonatología, con diagnóstico de sepsis el $10,3 \%(63 / 613)$, taquipnea transitoria del recién nacido $1 \%(6 / 613)$, enfermedad de la membrana hialina $1,6 \%$ $(10 / 613)$ y otras causas; el 99,3\% (609/613) fueron dados de alta y el $0,7 \%$ (4/613) fallecieron. Zhu et al (25) estudiaron a 10 neonatos hospitalizados, falleció el 10\% (1/10), 50\% (5/10) 
Revista del Cuerpo Médico del HNAAA, Vol 14 (3) - 2021

fueron dados de alta y 40\% (4/10) al momento del estudio continuaban hospitalizados, todos tuvieron prueba negativa para SARS-CoV-2. Breslin et $\mathrm{al}^{(27)}$ reportan una serie de 18 neonatos hijos de madre COVID-19 positivas, el $17 \%(3 / 18)$ fueron hospitalizados por otras razones: prematuridad, sepsis y riñón multiquístico congénito, siendo dados de alta posteriormente.

El porcentaje de muerte perinatal fue de 3,06\% (16 óbitos y 3 muertes neonatales temprana), Di Mascio et al..$^{(9)}$ en una revisión sistemática, reportan una tasa de muerte perinatal de $7 \%$ ( 2 de $41,95 \% \mathrm{Cl}, 1,4-16,3)$ incluye 1 natimuerto $(2,4 \%)$ y una muerte neonatal $(2,4 \%)$.

La prueba de PCR-RT tomada de faringe, dentro de las dos primeras horas de nacido, fue positiva en un $1,3 \%(8 / 613)$, de ellos $3 / 8$ fueron hospitalizados por presentar síntomas como taquipnea, vómitos, la evolución fue favorable y fueron dados de alta en buenas condiciones. 5/8 fueron recién nacidos a término, asintomáticos que permanecieron en alojamiento conjunto con sus madres y fueron dados de alta en buenas condiciones. Yu et al. ${ }^{(26)}$ reportó que a $3 / 7$ neonatos se les tomó la muestra para SARS-CoV-2, un paciente resultó positivo en la muestra tomada de hisopado faríngeo a las 36 horas de nacido, los otros dos fueron negativos. El neonato infectado presentó dificultad respiratoria leve y la radiografía de tórax mostró afectación pulmonar leve. Evolucionó favorablemente y fue dado de alta a las 2 semanas. El paciente tuvo SARS-CoV-2 a las 36 horas, sin embargo, la prueba de placenta y sangre de cordón umbilical fue negativa, por lo cual se pone en duda que haya sido una infección vertical. Así mismo Breslin et $\mathrm{al}^{(27)}$ reportan a 18 neonatos a los que se le tomó hisopado faríngeo para PCR-RT, el $84 \%(15 / 18)$ tuvieron resultado negativo en el día cero de nacidos, $11 \%(2 / 18)$ tuvieron resultado incierto y se les repitió la prueba el día 1 y 2 teniendo resultados negativos y $5 \%(1 / 18)$ tuvo resultado indeterminado.

En las características de los neonatos con PCR-RT positiva para SARS- CoV-2, en todos los casos la prueba se tomó hasta los 120 minutos de nacidos, $6 / 8$ (75\%) nacieron por cesárea y $2 / 8(25 \%)$ nacieron por vía vaginal, $2 / 8$ (25\%) presentaron sintomatología clínica: taquipnea y vómitos, con grado de severidad leve y $6 / 8$ (75\%) fueron asintomáticos, todos los casos salieron de alta vivos en buenas condiciones. Ma et $\mathrm{al}^{(28)}$ reportan 6 casos de neonatos a término, afectados por COVID-19, todos presentaron sintomatología leve, inespecífica en las primeras 36 horas de nacidos, caracterizada por fiebre, letargia, vómitos y fueron dados de alta recuperados. Bwire et $\mathrm{al}^{(29)}$ realizaron una revisión sistemática reportando 205 neonatos nacidos de madres con COVID-19, 13/205 $(6,3 \%)$ tuvieron pruebas positivas para SARS- CoV-2 al nacer. Sin embargo, Liguoro et $\mathrm{al}^{(30)}$ reportan que de 25 recién nacidos con SARS-CoV-2, hijos de madres con COVID-19, el12\% presentó enfermedad grave, siendo los signos más frecuentes la disnea, fiebre e intolerancia a la alimentación, el $20 \%$ presentaron cuadro clínico moderado y $20 \%$ leve. Siendo asintomáticos el $20 \%$ de casos.

En la tabla 9 se puede evidenciar que el número total de 16 óbitos, el $62,5 \%$ fueron extra hospitalarios y $37,5 \%$ intrahospitalario esto probablemente a que en nuestra realidad actualmente se ha hecho más complicado el acceso a servicios de salud en la gestante, como lo describe UNICEF en su nuevo reporte denominado Una tragedia olvidada: La carga mundial de la mortalidad fetal donde advierte que la pandemia de COVID-19 podría empeorar la tasa mundial de mortalidad fetal ${ }^{(31)}$. La reducción del $50 \%$ de los servicios de salud causada por la pandemia podría provocar cerca de 200.000 nuevas muertes fetales en un periodo de 12 meses en 117 países de ingresos bajos y medios. Esta cifra corresponde a un aumento del $11,1 \%$ de la carga de la mortalidad fetal. Tal como De Curtis y Villani encontraron que a pesar del triple aumento de mortinatos observado por ellos en comparación con el año anterior no les parece una consecuencia directa de la infección por COVID-19. Creen que podría ser una consecuencia de los cambios de vida inducidos por el encierro y, en particular, causada por la reducción de las visitas a los hospitales por temor a contraer la infección COVID-19. Este comportamiento ha llevado al aplazamiento o suspensión de todos los controles médicos incluso en mujeres embarazadas $^{(32)}$.

Stowe y Smith no encontraron evidencia de ningún aumento de mortinatos a nivel regional o nacional durante la pandemia de COVID-19 en Inglaterra ${ }^{(33)}$.

Otro hallazgo que tuvimos en nuestro estudio fue que el $93 \%$ de óbitos fueron en pacientes COVID-19 asintomático leve tal como encontraron Richtmann y Torloni en Brasil. En este estudio reportan 5 casos con muertes fetales y las cinco mujeres eran pacientes ambulatorias con formas leves o moderadas de COVID-19 y no tomaban ningún medicamento $^{(34)}$. Además, realizaron un estudio patológico placentario donde encontraron una intensa reacción inflamatoria placentaria en los cinco casos por lo que plantearon la posibilidad de un efecto directo del SARS-CoV-2 sobre la placenta, acto que no pudimos realizar debido a las limitaciones del servicio de patología de nuestro nosocomio, dejando la probabilidad que en nuestras pacientes también pudo ocurrir lo mismo.

De los resultados se puede concluir que el SRARS-CoV-2 adquirido por la madre en el tercer trimestre de gestación, no produce complicaciones severas en el neonato y que en los 8 casos que se obtuvo la prueba positiva de PCR-RT tomada de faringe ninguno presentó sintomatología grave.

\section{REFERENCIAS BIBLIOGRÁFICAS}

1. Actualización de la estrategia frente a la covid-19. Ginebra Suiza: OMS. Acceso 24 de junio 2020. disponible en h t t p s: / / w w w. who. int / docs / default source / coronaviruse / covid-strategy-update14april2020.pdf?sfvrsn=29da3ba0_19.

2. Terpos E, Ntanasis-Statoupoulos I, Elalamy I, Kastritis E, Sergentanis TN, Politou M, et al. Hematological finding and complications of COVID-19. Am J Hematol. 2020;: p. 1-14.

3. Farmacéuticos CGdC. Informe técnico corona-virus covid 19. Portalfarma. 2020;: p. 1-37. Acceso 24 de junio de 2020. D i s p o n i b l e e $n$ : https://www. mendeley.com/catalogue/81f898ea-3e5e-

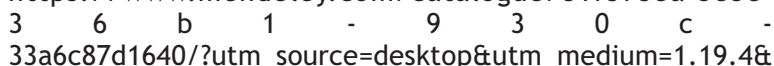
utm_campaign=open_catalog\&userDocumentld=\%7B57f89918 -4cb3-4de9-8dd9-f493c913ff00\%7

4. MINSA. Resolución MInisterial N 183-2021-MINSA. Acceso 24 de juniodel 202 . Disponible en: 
https: / / www.gob.pe/institucion/minsa/normas legales/1650082-183-2021-minsa.

5. Center CR. https://coronavirus.jhu.edu/map.html. [Online]; 2020. Acceso 24 de junio de 2020. Disponible en: https: / /coronavirus.jhu.edu/map.html.

6. MINSA. Covid en el Perú - Ministerio de Salud. [Online]; 2020. Acceso 24 de setiembrede 2020. Disponible en: https://covid19.minsa.gob.pe/sala_situacional.asp.

7. Gobierno Regional La Libertad, Sala situacional Covid 19. [Online]; 2020. Acceso 02 de 03de 2021. Disponible en: GRLaLibertad/posts/3478764378813215.

8. Marañón CT, Mastrapa CK, Poulut DTM, Vaillant LLD. COVID 19 y embarazo: una aproxsimación en tiempos de pandemia. MEDISAN (Internet). 2020; 4(24): p. 707-727.

9. Di Mascio D, Khalil A, Saccone G, Rizzo G, Buca D, Liberati M, et al. Outcome of coronavirus spectrum infections (SARS, MERS, COVID-19) during pregnancy: a systematic review and metaanalysis. Am J Obstet Gynecol MFM. 2020; 2(2): p. 100-107.

10. Manzini JL. Declaración de Helsinki: Principios éticos para la investigación médica sobre sujetos humano; Análisis de la 5ta reforma, aprobada por la Asamblea General de la Asociación Médica Mundical en octubre del año 2000, en Edimburgo. Acta Bioethica 2000. VI;(2): p. 321-334

11. Huerta Saenz IH, Elias Estrada JC, Campos del Castillo K, Muños Taya R, Coronado JC. Características materno perinatales de gestantes COVID-19 en un hospital nacional de Lima, Peru. Rev Peru Ginecol Obstet. 2020; 66(2).

12. Burga A. Estrategias de abordaje de la pandemia de covid-19 La Libertad al 06 de agosto 2020. Presentación de acciones de la oficina de Epidemiología e Información GERESA La Libertad. Acceso 24 de junio del 202.Disponible en: http://www.dge.gob.pe/portal/docs/tools/teleconferencia/ 2020/SE322020/02.pdf

13. Epidemiología CNd. Prevención y control de enfermedades. [Online]; 2020. Acceso el 06 de agosto del 202. Disponible en: https://www.dge.gob.pe/portal/index.php?option=com_con tent\&view=article\&id $=14$.

14. Mendoza M, García Ruiz I, Maiz N, Rodo C, García Manau P Serrano B, et al. Preeclampsia-like syndrome induced by severe COVID-19: a prospective observational study. BJOG 2020. Oct;127(11):1374-1380. doi: 10.1111/1471-0528.16339.

15. Lokken EM, Huebner EM, Taylor G, Hendrickson S, Vanderhoeven J, Kachikis A, et al. Disease severity, pregnancy outcomes, and maternal deaths among pregnant patients with severe acute respiratory syndrome coronavirus 2 infection in Washington State. Am J Obstet Gynecol. 2021.

16. Liu D, Li L, Wu X, Zheng D, Wang J, Yan L, et al. Pregnancy and Perinatal Outcomes of Women With Coronavirus Disease (COVID-19) Pneumonia: A Preliminary Analysis. AJR Am J Roentgenol. 2020; 215(1): p. 127-132.

17. Yan J, Guo J, Fan C, Juan J, Yu X, Li J, et al. Coronavirus disease 2019 in pregnant women: a report based on 116 cases. Am J Obstet Gynecol. 2020; 223(1).

18. Qiancheng X, Jian S, Lingling P, Lei H, Xiaogan J, Weihua L, et al. Coronavirus disease 2019 in pregnancy. Int J Infect Dis. 2020; 95: p. 376-383.

19. Chen N, Zhou M, Dong X, Qu J, Gong F, Han Y, et al. Epidemiological and clinical characteristics of 99 cases of 2019 novel coronavirus pneumonia in Wuhan, China: a descriptive study. The Lancet. 2020; 395(10223): p. 507-513.

20. Juan J, Gil M, Rong Z, Zhang Y, Yanhg H, Poon L. Effects of coronavirus disease 2019 (COVID-19) on maternal, perinatal and neonatal outcomes: a systematic review. Ultrasound Obstet Gynecol. 2020; 56(1): p. 15-27.

21. Schwartz DA. An Analysis of 38 Pregnant Women with COVID-19, Their Newborn Infants, and Maternal-Fetal Transmission of SARS-CoV-2: Maternal Coronavirus Infections and Pregnancy Outcomes. Arch Pathol Lab Med. 2020; 144(7): p. 799-805.

22. Gonzalez AO. Nefrología al día. [Online]; 2020. Disponible en: https: / / www.nefrologiaaldia.org/es-articulo-insuficienciarenal-aguda-infeccion-por-340.

23. Yang $H$, Sun G, Tang F, Peng M, Gao Y, Peng J, et al. Clinical features and outcomes of pregnant women suspected of coronavirus disease 2019. J Infect. 2020; 81(1): p. e40-e44.

24. Chen H, Guo J, Wang C, Lou F, Yu X, Zhang W, et al. Clinical characteristics and intrauterine vertical transmission potential of COVID-19 infection in nine pregnant women: a retrospective review of medical records. Lancet. 2020; 395(10226): p. 809-815.

25. Zhu H, Wang L, Fang C, Peng S, Zhang L, Chang G, et al. Clinical analysis of 10 neonates born to mothers with 2019-nCoV pneumonia. Transl Pediatr. 2020; 9(1): p. 51-60.

26. Yu N, Li W, Kang Q, Xiong Z, Wang S, Lin X, et al. Clinical features and obstetric and neonatal outcomes of pregnant patients with COVID-19 in Wuhan, China: a retrospective, single-centre, descriptive study. Lancet Infect Dis. 2020; 20: p. 559-64.

27. Breslin N, Baptiste C, Gyamfi-Bannerman C, Miller R, Martinez $\mathrm{R}$, Bernstein K, et al. COVID-19 infection among asymptomatic and symptomatic pregnant women: Two weeks of confirmed presentations to an affiliated pair of New York City hospitals. Am J Obstet Gynecol MFM. 2020; 2(2).

28. Ma X, Zhu J, Du L. Neonatal Management During the Coronavirus Disease (COVID-19) Outbreak: The Chinese Experience. Neoreviews. 2020; 21(5): p. e293-e297.

29. Bwire GM, Nijo BJ, Mwakawanga D, Sabas D, Sunguya BF. Possible vertical transmission and antibodies against SARSCoV-2 among infants born to mothers with COVID-19: a living systematic. J Med Virol. 230231; 93(3): p. 1361-1369.

30. Liguoro I, Pilotto C, Bonanni M, Ferrari ME, Pusiol A, Nocerino A, et al. SARS-COV-2 infection in children and newborns: a systematic review. Eur J Pediatr. 2020;: p. 1-18.

31. A neglected tragedy : the global burden of stillbirths. Report of the UN Inter-agency Group for Child Mortality Estimation. UNICEG-WHO-WBG-United Nations

32. De Curtis M, Villani L, Polo A. Increase of stillbirth and decrease of late preterm infants during the COVID-19 pandemic lockdown. Arch Dis Child Fetal Neonatal Ed. 2020.

33. Stowe J, Smith H, Trurland K, Ramsay ME, Andrews N, Landhani SN. Stillbirths During the COVID-19 Pandemic in England. JAMA. 2021; 325(1): p. 56-57.

34. Richtmann R, Torloni MR, Oyamada Otani AR, Levi JE, Tobara MC, Silva CdA, et al. Fetal deaths in pregnancies with SARSCoV-2 infection in Brazil: A case series. Case Reports Women's Heal. Case Rep Womens Health. 2020; 27 